Disturbi del sonno

\section{Consigli pratici di gestione nei pazienti eleggibili alle cure palliative pediatriche}

\author{
Irene Avagnina, Serena Condemi, \\ Chiara Paolin, Franca Benini
}

Centro Regionale Veneto di Terapia del Dolore e Cure Palliative Pediatriche, Dipartimento di Salute della Donna e del Bambino, Università di Padova

\section{Introduzione}

Le cure palliative pediatriche (CPP) rappresentano un ambito multidisciplinare della pediatria che si prefigge l'obiettivo di garantire la qualità di vita a bambini affetti da malattie inguaribili ad alta complessità assistenziale e di rispondere ai loro bisogni e a quelli delle loro famiglie.

A livello clinico, molteplici sono i sintomi disturbanti con ampia ricaduta sulla qualità della vita, che devono essere gestiti e trattati. Alcuni di questi in modo particolare rappresentano una sfida importante per i caregiver e i sanitari che si occupano dei bambini eleggibili alle CPP.

In questo quinto lavoro e nei successivi verranno affrontati il prurito e i disturbi del sonno, frequenti e invalidanti nei bambini in CPP.

\section{Disturbi del sonno}

\section{Definizione e cause}

Si definiscono "disturbi del sonno" quei disturbi che causano o un'alterata durata del sonno (deficit quantitativo) o un sonno disturbato da risvegli (deficit qualitativo).

Nei bambini eleggibili alle cure palliative pediatriche, i disturbi del sonno sono un sintomo molto frequente, con una prevalenza stimata tra il 5o e l'80\%, di cui i più colpiti sono i bambini con compromissione neurologica e sensoriale $(75-80 \%$ dei casi) [1-3]. Tale sintomo ha un impatto significativo sulla qualità di vita dei bambini e dei loro caregiver.

Secondo l'International Classification of Sleep Disorders (ICSD-3) i disturbi del sonno si possono categorizzare in:

1. insonnia: difficoltà a iniziare o mantenere il sonno;

2. disturbi del ritmo sonno-veglia;

3. ipersonnia;

4. disturbi del respiro correlati al sonno;

5. disturbi del movimento correlati al sonno.

In una recente casistica tedesca di 70 bambini e adolescenti seguiti dalle CPP, il $46 \%$ manifestava uno o più disturbi del sonno: il $94 \%$ dei casi mostravano insonnia, l' $84 \%$ disturbi del ritmo sonno-veglia, il 3I\% ipersonnia $[1,3]$.

I disturbi del sonno mostrano una prevalenza maggiore nei bambini affetti da:

- paralisi cerebrali infantili (>90\%);

- altre malattie con interessamento neurologico (malattie neuromuscolari e traumatiche);

- sindromi specifiche (Angelman, Prader-Willi, Rett, Williams, Cri du chat, Smith Magenis, Cornelia de Lange, Down).

La prevalenza è tuttavia elevata (>50\%) anche nelle altre patologie frequenti nei bambini seguiti dalle CPP (malattie metaboliche, oncologiche).
È stato inoltre osservato che maggiori sono le comorbidità rispetto alla patologia di base, maggiore è la probabilità di sviluppare un disturbo del sonno [2].

Tra i fattori eziologici troviamo:

- patologie di base (patologie neurologiche, disturbi sensoriali) e comorbidità associate (dolore, ingombro secretivo, spasticità, crisi epilettiche, disturbi del movimento, deprivazione sensoriale, reflusso gastroesofageo, ipossia, dispnea, apnee);

- fattori iatrogeni: farmaci (oppioidi, antiepilettici, salbutamolo), terapia a orario notturno, allarmi del monitoraggio (saturimetro), ventilazione meccanica notturna (soprattutto nel caso di interfaccia inadeguata o ventilazione non ottimale) [4];

- fattori ambientali (setting del sonno, ospedalizzazioni frequenti);

- fattori psicosociali (paura, stress, relazione con i caregiver, isolamento).

\section{Sintomatologia}

La riduzione della qualità del sonno determina una riduzione della qualità della veglia diurna, che può manifestarsi con sonnolenza e irritabilità, ma anche con un peggioramento della patologia di base (es. riduzione della soglia epilettica, aumento del distress o riduzione dei processi rigenerativi) e un peggioramento delle abilità cognitive (riduzione della relazione, del coping, dell'attenzione e del rendimento). Inoltre, sempre più evidenze scientifiche evidenziano la correlazione tra dolore e qualità del sonno, mostrando come lo scarso controllo di uno dei due sintomi determini un peggioramento/scarso controllo anche dell'altro. Quando il disturbo del sonno è inveterato può essere di difficile trattamento e può portare a serie conseguenze quali depressione, alterazione dei rapporti familiari, insorgenza di disturbi dell'umore. Inoltre, la persistenza del sintomo può portare anche ad alterazione delle performance fisiche e cognitive del caregiver, depressione e disturbi dell'umore anche nei familiari.

\section{Diagnosi}

La diagnosi dei disturbi del sonno è prevalentemente clinica e prevede l'inquadramento della tipologia di disturbo, la definizione dell'entità del sintomo e le sue ripercussioni sulla vita quotidiana.

A livello anamnestico le domande da porre sono:

- Quante ore dorme di notte? A che ora va a letto e a che ora si sveglia al mattino?

- Il bambino fa fatica ad addormentarsi?

- Quante volte si sveglia durante la notte? E perché si sveglia (Dolore? Crisi epilettiche? Difficoltà respiratoria? Necessità di posturazione?)

- I risvegli notturni sono disturbanti o il bambino si riaddormenta subito?

- Ci sono sintomi associati al disturbo del sonno, come difficoltà respiratoria, russamento, apnee del sonno, dolore?

- Al risveglio si associa agitazione, spavento o movimenti particolari? I risvegli sono sempre alla stessa ora?

- Il sonno appare riposante o il bambino si sveglia stanco?

- Durante il giorno è sonnolento? Quante ore dorme durante il giorno?

A completamento anamnestico può essere utile chiedere al caregiver un diario del sonno di alcuni giorni e una documentazione iconografica (video) soprattutto in caso di risvegli agitati o alterazioni nel sonno del pattern respiratorio.

È importante valutare il percepito del paziente e del caregir ver sulla qualità del sonno. Per questo esistono delle scale di valutazione validate che possono aiutare il clinico nell'inquadramento del sintomo: tra quelli usati e validati troviamo: la Sleep Disturbance Scale (SDSC), validata in età pedia- 
trica [5], la scala PROMIS [6] nel paziente oncologico, la scala SNAKE [7], rivolta a indagare la qualità del sonno nei bambini con compromissione neurologica. Tutte queste scale valutano aspetti sia qualitativi sia quantitativi del sonno con la differenza che le scale SDSC e SNAKE vengono compilate unicamente dal caregiver, mentre la PROMIS viene proposta sia al caregiver sia direttamente al paziente se di età superiore agli 8 anni. Inoltre, le scale SNAKE e PROMIS sono rivolte a popolazioni a rischio maggiore di disturbi del sonno (pazienti con compromissione neurologica e pazienti oncologici) e richiedono al momento della compilazione di far riferimento all'andamento del sonno nella settimana precedente. La scala SDSC invece è una scala validata per l'età pediatrica e prevede al momento della compilazione che si faccia riferimento ai 6 mesi precedenti, questo perché nella popolazione pediatrica generale i disturbi del sonno non sono così frequenti e un tempo di analisi maggiore permette di differenziare tra disturbi persistenti o transitori.

Infine, per la valutazione del sonno, soprattutto nei bambini con compromissione neurologica, è fondamentale considerare il riferito del caregiver e il burden complessivo del sintomo sul nucleo familiare [8].

All'esame obiettivo è bene valutare accuratamente l'esame neurologico completo, l'assessment del dolore e la valutazione del cavo orale e respiratoria.

Gli accertamenti strumentali sono indicati in caso si sospettino patologie neurologiche, respiratorie o dolore. La polisonnografia che prevede la registrazione simultanea dell'elettroencefalogramma, dell'elettrooculogramma e dell'elettromiografia associata alla registrazione dei parametri vitali notturni (saturimetria, frequenza cardiaca e respiratoria, ed eventualmente capnometria) è uno degli accertamenti più utili per la valutazione del sonno e permette di osservare anomalie specifiche, quali riduzione di numero e quantità delle fasi REM, riduzione dei movimenti oculari, aumento della fasi 4, anomalie dei fusi del sonno, presenza di pattern elettrici e respiratori anomali.

\section{Trattamento}

Il trattamento dei disturbi del sonno prevede [9-11]:

1. Trattamento delle cause sottostanti (dolore, crisi epilettiche, distonie, RGE).

2. Una corretta igiene del sonno, che si ottiene mediante l'applicazione delle seguenti regole:

- predisporre un setting adeguato all'addormentamento (far addormentare il bambino nella sua stanza, controllando la temperatura, la luce, i rumori ambientali; da preferire un ambiente buio, silenzioso, fresco, accogliente); - pianificare il ritmo circadiano (definire gli orari del sonno e di addormentamento/risveglio secondo quanto indicato per età e mantenerli nel tempo, evitare pisolini pomeridiani eccessivi);

- definire una routine dell'addormentamento (creare una ritualità dell'addormentamento costante nel tempo e che possa essere gestita anche in autonomia dal bambino);

- rispettare una routine quotidiana (orario dei pasti fisso, evitare sforzi o esercizi intensi prima del sonno, prediligere attività rilassanti prima dell'addormentamento);

A queste indicazioni possono essere associate terapie cognitivo-comportamentali, di cui sicuramente quelle per il rilassamento e controllo di ansie/emozioni sono estremamente utili soprattutto negli adolescenti o nell'end of life di pazienti senza compromissione neurologica.

3. Farmacoterapia (tutti i farmaci sottoriportati, a eccezione del triptofano e della melatonina, vengono utilizzati come off-label per i disturbi del sonno in età pediatrica):

- Melatonina: 0,I mg/kg, almeno 2-3 mg/dose ( $\max 5 \mathrm{mg}$ ) da assumere 20-30 minuti prima di coricarsi. Esiste sia la formulazione chrono che andrebbe utilizzata esclusivamente prima dell'addormentamento, sia la formulazione fast che può essere utilizzata sia all'addormentamento sia al primo risveglio. La melatonina sarebbe da utilizzare in profilassi nei bambini con ipovisione severa per garantire il mantenimento del ritmo circadiano.

- Triptofano, integratori (Pisolino-tripto), I-2 $\mathrm{ml}$ alla sera.

- Antistaminici: idrossizina 0,5 - $\mathrm{mg} / \mathrm{kg} / \mathrm{dose}$ (max $2 \mathrm{mg} /$ $\mathrm{kg} /$ die o max 50-Ioo mg/die) in mono-somministrazione serale all'addormentamento o al primo risveglio (ripetibile fino a 4 volte/die). Pro: in caso di difficoltà di addormentamento o irritabilità. Effetti collaterali: prolungamento QT, sedazione.

- Niaprazina (Nopron sciroppo $3 \mathrm{mg} / \mathrm{ml}$ ): I mg/kg (max 30-60 mg), in mono-somministrazione serale all'addormentamento o al primo risveglio. Solitamente si inizia con metà dose il primo giorno e poi si incrementa in base alla tolleranza. Pro: nei disturbi del sonno con frequenti risvegli. Effetti collaterali: sonnolenza diurna, vomito, stipsi, eccitazione paradossa.

- Alfa-agonisti: clonidina dose 2-4 $\mathrm{mcg} / \mathrm{kg}$ alla sera per via orale; nei bambini più grandi iniziare con dosi non superiori ai IOO $\mathrm{mcg}(\max 400 \mathrm{mcg}$ o $8 \mathrm{mcg} / \mathrm{kg})$. Effetti collaterali: ipotensione ortostatica, sonnolenza, secchezza delle fauci, cefalea. Considerare anche in alternativa dexmedetomidina I.N. (I per $4 \mathrm{mcg} / \mathrm{kg} / \mathrm{die}$ ) soprattutto in caso di risvegli frequenti e invalidanti.

- Benzodiazepine: Zolpidem o,I-0,25 mg/kg (5-Io mg). Efficacia dubbia in pediatria. Effetti collaterali: cefalea, capogiro, allucinazioni.

- Antidepressivi: trazodone a basse dosi (0,75-I mg/kg; oppure 25-50 mg nei grandi fino a max I50 mg).

- Antipsicotici atipici.

- Anticonvulsivanti, fenobarbital in mono-somministrazione serale, soprattutto nei bambini con epilessia o quadri di neuroirritabilità.

Prima di iniziare una farmacoterapia vanno presi in considerazione vari aspetti, quali eventuali interazioni con altri farmaci, la malattia di base, l'età del bambino, il tipo di disturbo del sonno e la sua gravità [3].

\section{Take home message}

- I disturbi del sonno sono un sintomo molto frequente, che interessa più del 50\% dei bambini eleggibili alle CPP e determinano un significativo burden per le famiglie.

- Indagare la qualità del ritmo sonno-veglia è parte integrante della raccolta dei bisogni in CPP.

- I disturbi del sonno vanno adeguatamente inquadrati e trattati.

\section{Caso clinico}

Gianni ha 4 anni e pesa I $8 \mathrm{~kg}$; è affetto da distrofia muscolare e lieve ritardo psicomotorio. Per un quadro di insufficienza respiratoria acuta Gianni è stato recentemente ricoverato per I mese in terapia intensiva pediatrica ed è stato poi dimesso con una ventilazione non invasiva.

Dal rientro a domicilio i genitori riferiscono che Gianni fa fatica ad addormentarsi, prende sonno verso le 2 di notte ormai sfinito e rifiuta la ventilazione che i genitori avviano dopo che ha preso sonno. Inoltre vengono segnalati altri 2 risvegli notturni con difficoltà di riaddormentamento.

Viene proposto avvio di melatonina alle 2I, mezzora prima dell'addormentamento e viene rimodulata la ventilazione prevedendo un avvio progressivo nei primi 5 minuti di NIV. Con queste modifiche terapeutiche si assiste a un lieve miglioramento del sonno con un addormentamento verso le 23. Tuttavia Gianni continua a presentare risvegli notturni con difficoltà di riaddormentamento. Viene pertanto proposta tera- 
pia con niaprazina $\mathrm{I} 5 \mathrm{mg}$ da somministrare al primo risveglio. Con tale terapia Gianni dorme dalle 23:00 alle 7:00, con un riferito risveglio notturno breve per posturazione.

\section{Bibliografia}

1. Dreier LA, Zernikow B, Stening K, Wager J. Insights into the Frequency and Distinguishing Features of Sleep Disorders in Pediatric Palliative Care Incorporating a Systematic Sleep Protocol. Children (Basel). 202I Jan I7;8(I):54.

2. Tietze AL, Blankenburg M, Hechler T, et al. Sleep disturbances in children with multiple disabilities. Sleep Med Rev. $20 \mathrm{I2}$ Apr;I6(2):II7-I27.

3. Heussler HS, Hiscock H. Sleep in children with neurodevelopmental difficulties. J Paediatr Child Health. 20I8 Oct;54(IO):II42-II47.

4. Verrillo E, Pavone M, Bruni O, et al. Effects of long-term non-invasive ventilation on sleep structure in children with Spinal Muscular Atrophy type 2. Sleep Med. 20I9 Jun;58:82-87.

5. Bruni O, Ottaviano S, Guidetti V, et al. The Sleep Disturbance Scale for Children (SDSC). Construction and validation of an instrument to evaluate sleep disturbances in childhood and adolescence. J Sleep Res. 1996 Dec;5(4):25I-26I.

6. Daniel LC, Gross JY, Meltzer LJ, et al. Clinical validity of the PROMIS pediatric sleep short forms in children receiving treatment for cancer. Pediatr Blood Cancer. 2020 Sep;67(9):e28535.
7. Dreier LA, Zernikow B, Blankenburg M, Wager J. A Sleep Questionnaire for Children with Severe Psychomotor Impairment (SNAKE)-Concordance with a Global Rating of Sleep Quality. Children (Basel). 2018 Feb I;5(2):20.

8. Halstead EJ, Jones A, Esposito G, Dimitriou D. The Moderating Role of Parental Sleep Knowledge on Children with Developmental Disabilities and Their Parents' Sleep. Int J Environ Res Public Health. 202I Jan I6;I8(2):746.

9. McDonagh MS, Holmes R, Hsu F. Pharmacologic Treatments for Sleep Disorders in Children: A Systematic Review. J Child Neurol. 2019 Apr;34(5):237-247.

10. De Zen L, Del Rizzo I, Robazza M, et al. Home Use of Intranasal Dexmedetomidine in a Child With An Intractable Sleep Disorder. J Pediatr Pharmacol Ther. 2020;25(4):332-335.

11. Bruni o, Angriman M, Calisti F, et al. Practitioner Review: Treatment of chronic insomnia in children and adolescents with neurodevelopmental disabilities. J Child Psychol Psychiatry. 20I8 May;59(5):489-508.

\section{Farmaci sintomatici per raffreddore e tosse: tutt'altro che innocui}

\section{blister}

Negli anni 2007-2008, negli USA, l'FDA aveva posto sotto i riflettori il profilo di sicurezza di alcuni farmaci da banco, avendo realizzato che un bambino su due di età $<4$ anni, con sintomi di raffreddore o tosse, era trattato con prodotti di questo tipo [1]. Questa opera di revisione aveva portato a successivi provvedimenti restrittivi, fino alla raccomandazione di non utilizzare farmaci sintomatici per il raffreddore e la tosse (FSRT) in bambini di età <2 anni e all'istituzione di un osservatorio nazionale denominato Pediatric Cough and Cold Safety Surveillance System. L'anno successivo veniva pubblicata una casistica che raccoglieva i decessi causati dai FSRT: si trattava, in gran parte, di bambini di età <2 anni, sottoposti a un sovradosaggio, in alcuni casi, con un possibile intento lesivo. Gli autori di questo ulteriore lavoro hanno inteso indagare cosa fosse cambiato dal 2008 in poi (e fino al 2016) nel verificarsi di decessi conseguenti all'utilizzo di FSRT in bambini di età <12 anni [2]. I farmaci presi in considerazione erano 8: bromfeniramina, clorfeniramina, destrometorfano, difenidramina, doxilamina, guaifenesina, fenilefrina e pseudoefedrina. Su un totale di quasi 8000 segnalazioni di effetti collaterali potenzialmente riconducibili ai FSRT, $188(2,4 \%)$ erano stati mortali e, di questi, 40 risultavano con buona probabilità correlati a uno dei principi attivi considerati. La difenidramina risultava implicata in 28/40 (70\%) casi e in $11 / 40$ era in associazione con un oppioide. II 60\% aveva un'età <2 anni e solo in 3/40 (7,5\%) casi si è trattato di un'ingestione accidentale. In 22/40 (55\%) eventi mortali, la somministrazione del farmaco non aveva un chiaro intento terapeutico.

Volendo sintetizzare, possiamo dire che appaiono a elevato rischio di morte per FSRT soprattutto i bambini di età $<2$ anni, nei quali la somministrazione è avvenuta a opera dei genitori, spesso con intento non terapeutico, forse sedativo. In alcuni casi è stato possibile accertare l'intento lesivo. Difenidramina, clorfeniramina e destrometorfano sono i principi attivi maggiormente responsabili. Escludendo i casi di somministrazione dolosa, appare evidente la potenziale pericolosità di questi farmaci nei primi anni di età e la necessità di una grande consapevolezza nella prescrizione da parte dei medici e nella somministrazione non controllata da parte dei genitori.

1. Dart RC, Paul IM, Bond GR, et al. Pediatric fatalities associated with over the counter (nonprescription) cough and cold medications. Ann Emerg Med. 2009 Apr;53(4):4II-4I7.

2. Halmo LS, Wang GS, Reynolds KM, et al. Pediatric Fatalities Associated With Over-the-Counter Cough and Cold Medications. Pediatrics. 202I Nov;I48(5):e2020049536. 